\title{
Use of a Barbed Suture Tie-Over Technique for Skin Graft Dressings: A Case Series
}

\author{
Kenneth M Joyce ${ }^{1}$, Cormac W Joyce ${ }^{1}$, Nicola Mahon ${ }^{1}$, Jack L Kelly ${ }^{1,2}$ \\ ${ }^{1}$ Department of Plastic and Reconstructive Surgery, Galway University Hospital, Galway; ${ }^{2}$ Department of Surgery, National University of \\ Ireland, Galway, Ireland
}

Background A tie-over dressing is the accepted method to secure skin grafts in order to prevent haematoma or seroma formation. We describe the novel application of a barbed suture tie-over for skin graft dressing. The barbs act as anchors in the skin so constant tensioning of the suture is not required.

Methods From January 2014 to August 2014 we used the technique in 30 patients with skin defects requiring split-thickness or full-thickness grafts. Patient demographics, clinicopathological details and graft outcome were collected prospectively.

Results The majority of cases were carried out for split-thickness skin grafts $(n=19)$ used on the lower limb $(n=20)$. The results of this novel technique were excellent with complete $(100 \%)$ graft take in all patients.

Conclusions Our results demonstrate the clinical application of a barbed device for securing skin grafts with excellent results. We find the technique quick to perform and the barbed device easy to handle, which can be applied without the need for an assistant.

Keywords Skin transplantation / Occlusive dressings / Graft survival
Correspondence: Kenneth M Joyce Department of Plastic and Reconstructive Surgery, Galway University Hospital, Galway 0000, Ireland

Tel: +353879654586

Fax: +35391542222

E-mail: kennethjoyce1@gmail.com

No potential conflict of interest relevant to this article was reported.

\section{INTRODUCTION}

Skin grafting is the most commonly and easily utilized method in reconstructing soft tissue defects. One of the primary requirements for successful grafting is the application of even pressure to the graft by a carefully designed dressing, described by Blair and Brown [1] in 1929. The traditional method of skin graft stabilization is the tie-over technique or bolster dressing [2]. Previously reported methods of securing a skin graft include skin staples [3], rubber bands [4], negative pressure dressings [5], and fibrin glue [6] among many others. No consensus exists as to which is the optimum method.

The simple tie-over dressing is well renowned although many modifications of the classic technique have been described $[7,8]$. This technique has remained popular because of its accessibility and simplicity. However, in our experience, the classic tie-over is both time-consuming and laborious and the need for constant tensioning is often difficult to perform especially when operating without an assistant. Barbed sutures evenly distribute tension along the length of the suture and provide immediate tissue hold on placement, thereby eliminating the need for an assistant and negating the need for constant tensioning. No requirement of a knot facilitates a reduced operating time [9]. We have previously reported on a barbed tie-over technique on a cadaveric model and we now present this translated research in a case series of 30 patients. 


\section{METHODS}

This technique is carried out routinely by the senior author to stabilize skin grafts. We used a 3-0 polydioxanone Stratafix (Ethicon Inc., Somerville, NJ, USA) suture for this purpose. The suture is a bidirectional device with 20 barbs per centimeter of suture material. The barbs are distributed circumferentially around the suture at $120^{\circ}$ rotations and each bark measures $0.38 \mathrm{~mm}$ in length. There is a needle at each end of the suture and the barbs change direction at the midpoint.

A full thickness or split-thickness skin graft is harvested and laid onto the wound defect. The graft is sutured at the wound edges using interrupted 4-0 Vicryl Rapide sutures. A non-adherent dressing is applied using Mepitel and mineral-oil soaked wool. The running barbed technique is carried out by initially taking a small bite of skin at the 12 o' clock position of the graft and the suture is then pulled through until resistance is achieved (Fig. 1). This signifies the transition point at the centre of the device where the barbs change direction. This acts as an anchor for the suture device. The tie-over is then formed using the two ends of the barbed suture until sufficient stabilization has been formed. A controlled pull after each skin bite will apply downward pressure, which is sustained by the unidirectional barbs. No constant tensioning is required. The unidirectional nature of the barbs prevents any slippage and the barbs even act as a grip on the underlying wool dressing. At the end, the two needles are cut from the barbed suture. No knot is required. The tie-over dressing is removed easily in the dressing clinic, without any anaesthesia after an average of five days (range, 5- 8 days). The barbs are removed by cutting the suture at the transition point and puling the suture in the opposite direction of the barbs. This is easily

Fig. 1. Diagram representing the bidirectional barbed tie-over dressing

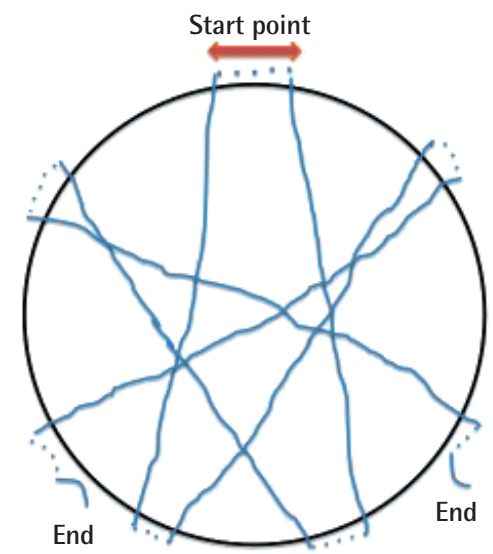

achieved as it is the only direction the barbs will pass without resistance.

\section{RESULTS}

Between January 2014 and August 2014 we used the barbed suture for tie-over dressing in 30 patients who had a skin defect that we reconstructed with either a split-thickness or full-thickness skin graft. The patient demographics and outcomes were recorded prospectively (Table 1 ). The majority of barb tie-overs were for split thickness skin grafts $(n=19)$ and the remaining for full thickness grafts $(\mathrm{n}=11)$, e.g., Fig. 2. The mean age was 67.4 years (range, 50-91 years). Of the 30 patients, the underlying pathology was skin malignancy in 22 patients, followed by chronic wounds $(n=4)$ and trauma $(n=4)$. The most frequently applied area was the lower $\operatorname{limb}(\mathrm{n}=20)$, e.g., Fig. 3 although we also used the barbed tie-over on the scalp $(n=5)$, neck $(n=2)$, and upper $\operatorname{limb}(\mathrm{n}=3)$. The average area of defect requiring graft was $37 \mathrm{~cm}^{2}$ (range, $9-150 \mathrm{~cm}^{2}$ ).

Patient's overall satisfaction with the tie-over dressing was good. We were initially concerned that the exerted pressure would have been too high for the graft leading to necrosis but we found that all the grafts took completely (100\%). No complications were observed. Our initial concerns were that the tie over would create suture marks in the surrounding skin although all these resolved completely over time.

\section{DISCUSSION}

Adequate stabilisation of a graft to the wound bed is imperative for successful graft take and in preventing complications such as haematoma or seroma formation. The classic tie-over dressing is cheap and simple. However, the act of tying the knot typically requires an assistant and can take an increased amount of time relative to the excision procedure [10]. Various alterations in the traditional tie-over have been described in an effort to maximize effective downward pressure, including twist-tie [10], silicone tubing [11] and loop threads [8]. This variation in methods reflect a lack in consensus in the optimum material and technique.

We find this novel technique quick to perform and the barbed device easy to handle. The unidirectional nature of each barb prevents any slippage and negates the need to apply constant pressure. It can be applied without the need for an assistant and therefore leads to more efficient use of theatre time. In all cases of this study, no haematoma, seroma or graft failure was observed and there was good patient satisfaction. By removing the sutures on average on the fifth postoperative day, both scar formation and local reactions to the suture material were prevented. 
Table 1. Patient demographics who underwent barbed suture tie-over dressing

\begin{tabular}{|c|c|c|c|c|c|c|}
\hline Patient no. & Age (yr) & Indication & Area & Graft & Graft take (\%) & Defect size $(\mathrm{cm})$ \\
\hline 1 & 52 & Malignancy & Scalp & FTSG & 100 & $4 \times 4$ \\
\hline 2 & 78 & Malignancy & Lower limb & FTSG & 100 & $6 \times 4$ \\
\hline 3 & 81 & Malignancy & Lower limb & FTSG & 100 & $4 \times 3$ \\
\hline 4 & 68 & Chronic wound & Lower limb & STSG & 100 & $5 \times 3$ \\
\hline 5 & 61 & Chronic wound & Lower limb & STSG & 100 & $4 \times 4$ \\
\hline 6 & 57 & Malignancy & Lower limb & STSG & 100 & $4 \times 3$ \\
\hline 7 & 59 & Malignancy & Scalp & STSG & 100 & $3 \times 3$ \\
\hline 8 & 71 & Malignancy & Lower limb & STSG & 100 & $15 \times 10$ \\
\hline 9 & 63 & Trauma & Lower limb & STSG & 100 & $8 \times 7$ \\
\hline 10 & 64 & Trauma & Lower limb & STSG & 100 & $12 \times 9$ \\
\hline 11 & 91 & Malignancy & Neck & FTSG & 100 & $5 \times 3$ \\
\hline 12 & 82 & Chronic wound & Lower limb & STSG & 100 & $12 \times 10$ \\
\hline 13 & 67 & Malignancy & Scalp & FTSG & 100 & $4 \times 3$ \\
\hline 14 & 62 & Trauma & Lower limb & STSG & 100 & $8 \times 6$ \\
\hline 15 & 78 & Malignancy & Lower limb & STSG & 100 & $9 \times 5$ \\
\hline 16 & 61 & Malignancy & Upper limb & STSG & 100 & $4 \times 3$ \\
\hline 17 & 50 & Malignancy & Neck & STSG & 100 & $6 \times 4$ \\
\hline 18 & 85 & Chronic wound & Lower limb & STSG & 100 & $8 \times 8$ \\
\hline 19 & 88 & Malignancy & Lower limb & STSG & 100 & $5 \times 5$ \\
\hline 20 & 70 & Malignancy & Upper limb & STSG & 100 & $3 \times 3$ \\
\hline 21 & 43 & Trauma & Lower limb & FTSG & 100 & $4 \times 4$ \\
\hline 22 & 78 & Malignancy & Lower limb & FTSG & 100 & $6 \times 3$ \\
\hline 23 & 69 & Malignancy & Lower limb & STSG & 100 & $3 \times 2$ \\
\hline 24 & 67 & Malignancy & Scalp & FTSG & 100 & $4 \times 3$ \\
\hline 25 & 81 & Malignancy & Lower limb & STSG & 100 & $7 \times 5$ \\
\hline 26 & 77 & Malignancy & Scalp & STSG & 100 & $7 \times 6$ \\
\hline 27 & 85 & Malignancy & Lower limb & FTSG & 100 & $8 \times 3$ \\
\hline 28 & 80 & Malignancy & Upper limb & FTSG & 100 & $4 \times 3$ \\
\hline 29 & 52 & Malignancy & Lower limb & STSG & 100 & $11 \times 5$ \\
\hline 30 & 88 & Malignancy & Lower limb & FTSG & 100 & $4 \times 4$ \\
\hline
\end{tabular}

\section{Fig. 2. Photograph of patient number 2}

(A) Wound defect in patient no. 2 post excision of skin malignancy with full-thickness skin graft in situ. (B) Barbed suture used to form tie-over dressing over mineral oil-soaked wool. (C) The full thickness graft in Figs. 1 and 2 two weeks post removal of the tie-over dressing.
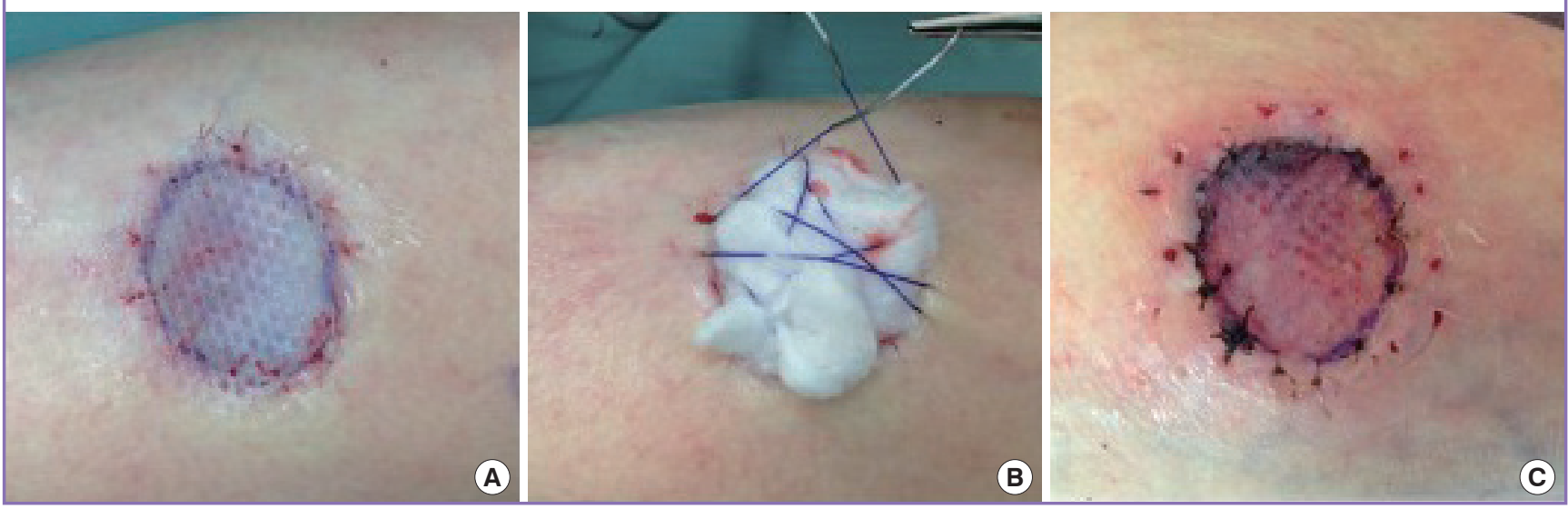

The authors acknowledge several limitations to the described technique. Firstly, the barbed suture is relatively expensive in comparison to conventional sutures. However, a classic silk tieover typically requires several silk sutures therefore the overall disparity in cost between the two methods is minimal. In theory, a minimum downward pressure of $25 \mathrm{~mm} \mathrm{Hg}$ is required by the tie-over dressing to exceed capillary pressure and prevent haematoma formation [12]. It has been proposed that pressure ne- 


\section{Fig. 3. Photograph of wound from patient number 10}

(A) Wound from patient no.10 following dog bite to lower leg. (B) Patient no.10 following debridement of wound lower leg. (C) Patient no. 10 with barbed tie over dressing in situ. (D) Graft review of patient no. 10 at two weeks.
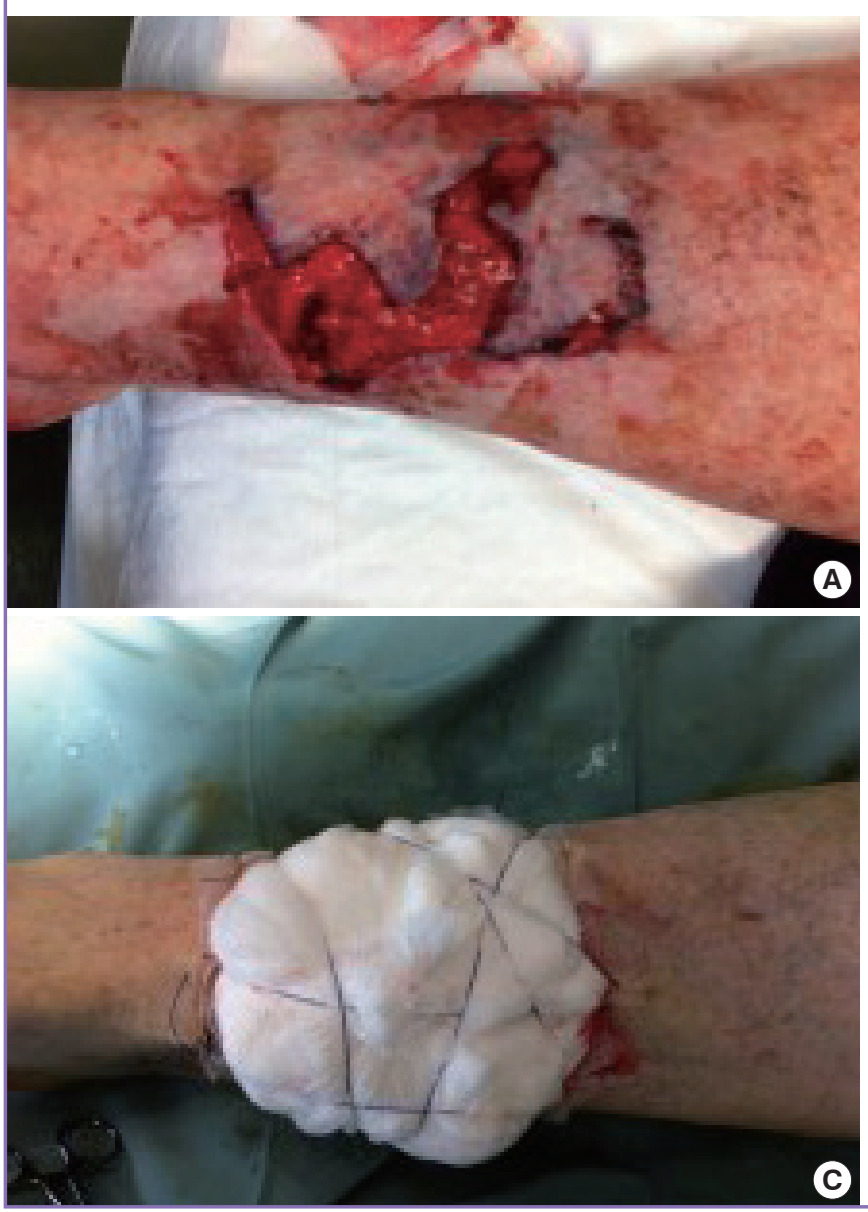

crosis may occur if too much pressure is exerted, especially over bony prominences [12]. However, negative pressure devices are frequently employed over skin grafts with pressures over 100 $\mathrm{mmHg}$ frequently used. No deleterious pressure effects have been reported with vacuum therapies on skin grafts. Previous laboratory studies from our institution demonstrated an improved downward pressure achieved by a barbed tie-over dressing compared to traditional methods [13]. Further studies are necessary to determine the necessary range of downward pressure required. The barbed suture requires adequate surrounding skin to anchor in order to achieve downward pressure and relative contra-indications include patients with thin skin (e.g., elderly or patients taking steroids). The use of barbs in these scenarios may create stitch sinuses or tearing in the surrounding skin. The authors recommend a smaller calibre barbed suture (e.g., 5-0/60 ) for application of this technique on the face.

We have previously demonstrated on a cadaveric model that barbed tie-overs are both quicker to perform and exert a higher downward pressure on the skin graft compared to traditional
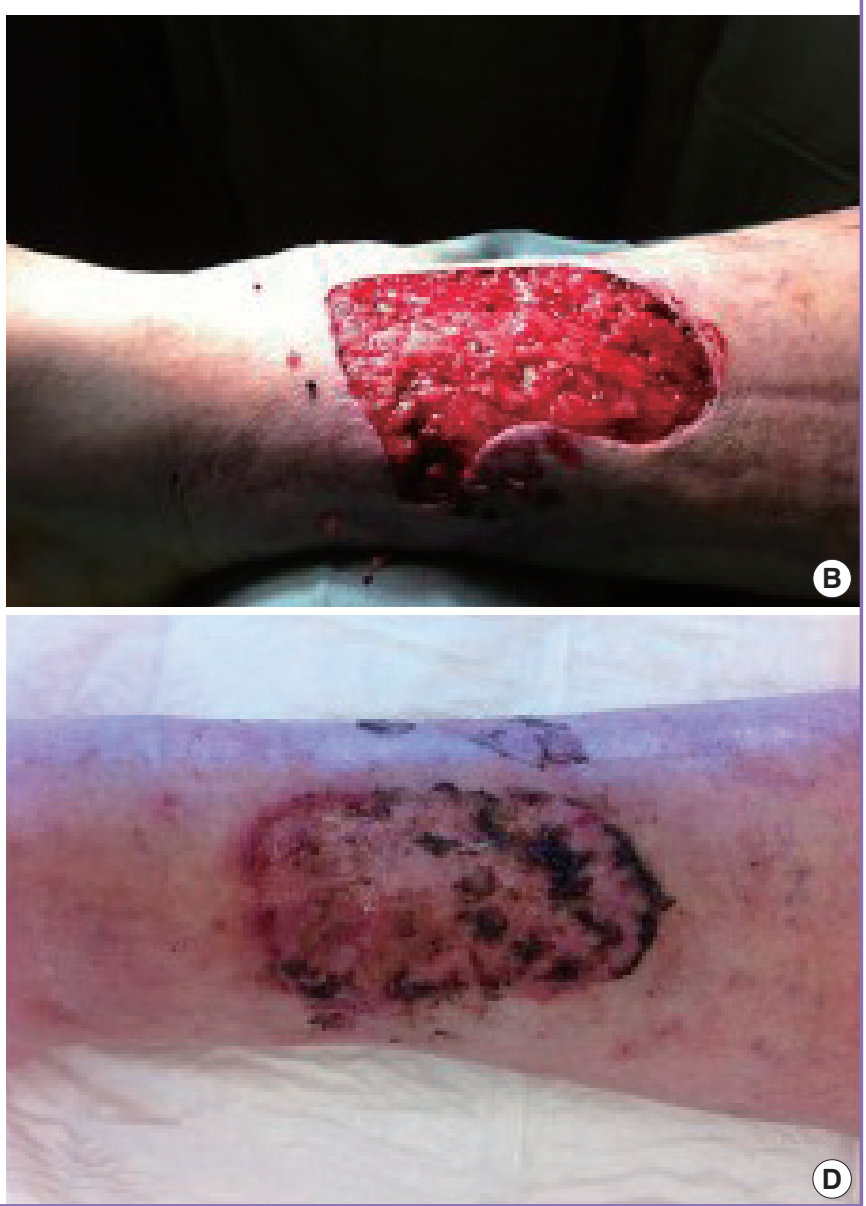

tie-over methods using silk sutures. We have shown that the barbed-tie over is twice as quick to perform than the silk tie-over (105 \pm 22 seconds vs. $266 \pm 39$ seconds, respectively) in a cadaveric model. Following on from this laboratory study, we now report on a clinical case series of 30 patients who had a barbed suture tie-over to secure their skin grafts. It is now the dressing of choice for securing skin grafts in our institution. We find this technique easy to use and it has produces consistently good results. Furthermore, the barbed tie-over saves on valuable operative time and the technique does not require an assistant. These added economic benefits will make it a very useful option in the plastic surgeon's armamentarium.

\section{REFERENCES}

1. Blair VP, Brown JB. The use and uses of large split skin grafts of intermediate thickness. Plast Reconstr Surg 1968;42:6575.

2. Davenport M, Daly J, Harvey I, et al. The bolus tie-over "pres- 
sure" dressing in the management of full thickness skin grafts. Is it necessary? Br J Plast Surg 1988;41:28-32.

3. Freeman BS. The use of the pocket stapler in surgery. Plast Reconstr Surg Transplant Bull 1962;30:289-91.

4. Fleury AL, Rees TD. The use of foam rubber in pressure dressings. Plast Reconstr Surg (1946) 1956;18:309-11.

5. Blackburn JH 2nd, Boemi L, Hall WW, et al. Negative-pressure dressings as a bolster for skin grafts. Ann Plast Surg 1998; 40:453-7.

6. Buckley RC, Breazeale EE, Edmond JA, et al. A simple preparation of autologous fibrin glue for skin-graft fixation. Plast Reconstr Surg 1999;103:202-6.

7. Niranjan NS. A modified tie-over dressing for skin grafts. $\mathrm{Br}$ J Plast Surg 1985;38:415-8.

8. Jo HJ, Kim JS, Kim NG, et al. Redoable tie-over dressing using multiple loop silk threads. Arch Plast Surg 2013;40:259-
62.

9. Grigoryants V, Baroni A. Effectiveness of wound closure with V-Loc 90 sutures in lipoabdominoplasty patients. Aesthet Surg J 2013;33:97-101.

10. Pleat JM, O’Reilly DJ, Cogswell LK, et al. The "twist over": tie die? Plast Reconstr Surg 2006;117:324-5.

11. Bektas CI, Kankaya Y, Ozer K, et al. A tie-over dressing using a silicone tube to graft deep wounds. Arch Plast Surg 2013;40:711-4.

12. Seymour FK, Giele HP. Tie-overs under pressure. Br J Plast Surg 2003;56:494-7.

13. Joyce CW, Joyce KM, Mahon N, et al. A novel barbed suture tie-over dressing for skin grafts: a comparison with traditional techniques. J Plast Reconstr Aesthet Surg 2014;67:123741. 